

\section{REVISTA \\ TEORÍA Y PRÁCTICA \\ DE LA \\ ARQUEOLOGÍA HISTÓRICA LATINOAMERICANA}

ISSN: 2250-866X (impreso) | ISSN: 2591-2801 (en línea)

AÑo VIII, VOLUMEN 8, PRIMAVERA DE 2019

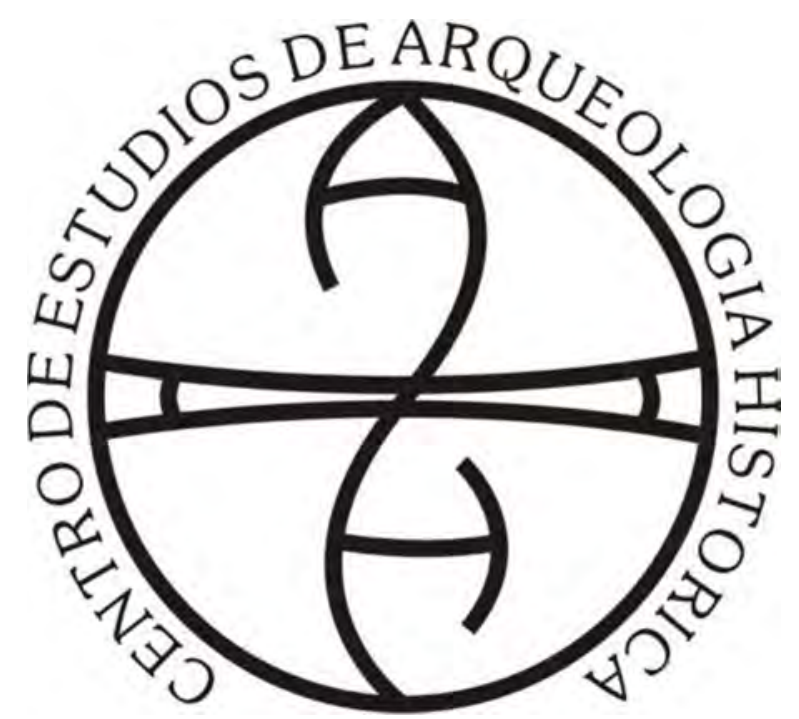

Centro de Estudios de Arqueología Histórica

FACULTAD DE HUMANIDADES Y ARTES | UNIVERSIDAD NACIONAL DE ROSARIO 
(Universidad Nacional de Rosario, Universidad Nacional de Río Cuarto,

Universidad Nacional de San Juan, Universidad de la República, Universidad Nacional de Trujillo)

\author{
AUTORIDADES DE LA UNIVERSIDAD NACIONAL DE ROSARIO \\ RECTOR: Lic. Franco Bartolacci \\ VICE-RECTOR: Od. Darío Macía \\ SECRETARIO GENERAL: Prof. José Goity \\ SECRETARIA ACADÉMICO Y DE APRENDIZAJE: Dr. Marcelo Vedrovnik \\ SECRETARÍA DE CIENCIA TECNOLOGÍA E INNOVACIÓN \\ PARA EL DESARROLLO: Ing. Guillermo Montero.
}

\author{
AUTORIDADES DE LA FACULTAD DE HUMANIDADES Y ARTES \\ DECANO: Prof. Alejandro Vila \\ VICEDECANA: Prof. Marta Varela \\ SECRETARIA ACADÉMICA: Dra. Marcela Coria
}

\author{
AUTORIDADES DEL CENTRO DE ARQUEOLOGÍA HISTÓRICA \\ DIRECTORA: Dra. Ana Rocchietti \\ SECRETARIA: Prof. Nélida de Grandis \\ PROSECRETARIA: Lic. Marianela Bizcaldi
}

DIRECTORAS - EDITORAS:

Dra. Ana Rocchietti y Prof. Nélida De Grandis

SECRETARIA DE EDICIÓN: Dra. Irene Dosztal

Este número es co-edición de las ponencias

del VIII Congreso Nacional de Arqueología Histórica (2018) entre:

Centro de Estudios en Arqueología Histórica: Directora Ana Rocchietti

Centro de Estudios en Arqueología Regional: Director Fernando Oliva

Centro de Estudios en Arqueología Subacuática: Directora Mónica Valentini

Departamento de Arqueología, Escuela de Antropología: Director Fernando Oliva
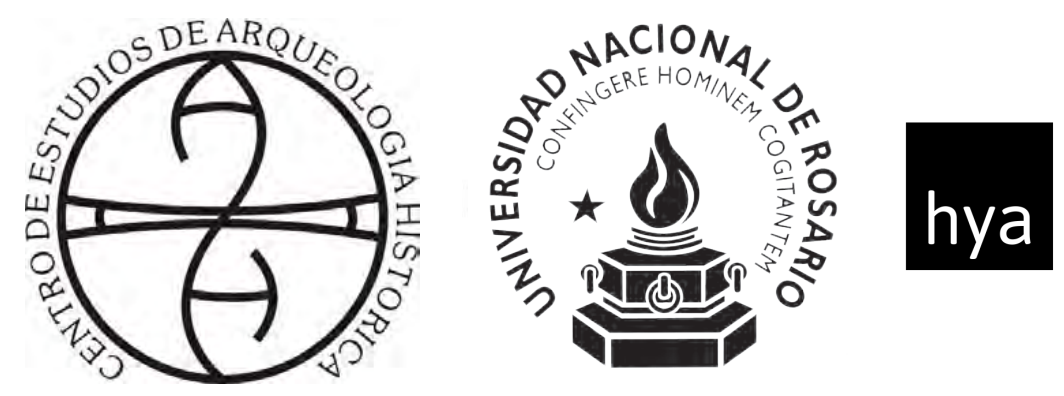

Facultad de Humanidades

y Artes_UNR
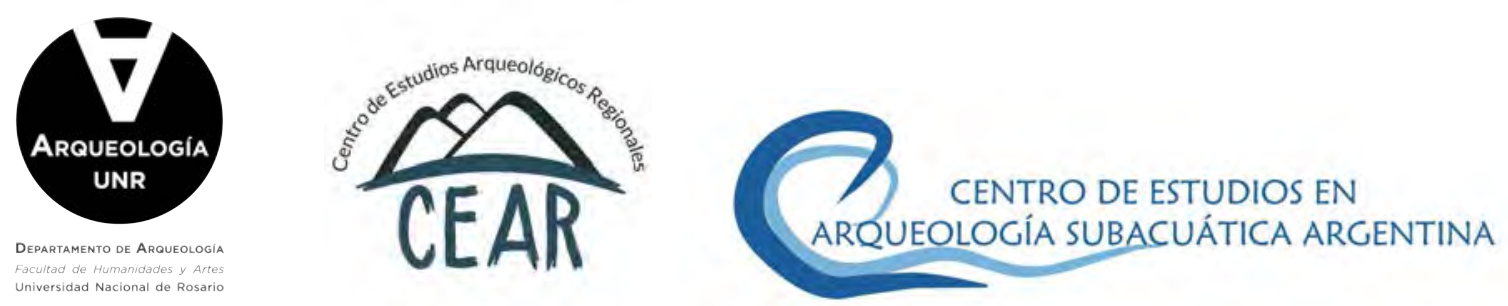
Comité Permanente de los Congresos Nacionales de Arqueología Histórica

Dr. Daniel Schávelzon (Universidad Nacional de

Buenos Aires)

Prof. María Teresa Carrara (Universidad Nacional de Rosario)

Prof. Carlos Baldassarre (Museo Municipal de Río

Grande, Tierra del Fuego) in memoriam

Dr. Mariano Ramos (Universidad Nacional de Luján, CONICET)

Dr. Horacio Chiavazza (Universidad Nacional de Cuyo)

Dra. Ana María Rocchietti (Universidad Nacional de

Rosario, Universidad Nacional de Río Cuarto)

Lic. Facundo Gómez Romero (Universidad Autónoma

de Barcelona)

\section{Comité Científico}

Dra. Tânia Andrade Lima (Universidade Federal do Rio de Janeiro)

Prof. Réginald Auger (CELAT/Département des

Sciences Historiques, Université Laval, Canadá)

Dr. Roberto Bárcena (Universidad Nacional de Cuyo, CONICET)

Dra. Marta Bonaudo (Universidad Nacional de Rosario, CONICET)

Dr. Leonel Cabrera (Universidad de la República, Uruguay)

Dr. Luis María Calvo (Universidad Católica de Santa

$\mathrm{Fe})$

Prof. Juan Castañeda Murga (Universidad Nacional de

Trujillo, Perú)

Dr. Carlos Ceruti (Museo de Ciencias Naturales y

Antropología "Prof. Antonio Serrano". Paraná)

Dr. Horacio Chiavazza (Universidad Nacional de Cuyo)

Dra. Silvia Cornero (Universidad Nacional de Rosario)

Prof. Pedro Paulo Funari (Universidade Estadual de

Campinas, Brasil)

Lic. Jorge A. Gamboa Velásquez (Universidad Nacional

Santiago Antuñez de Mayolo, Perú)

Dr. Eduardo Alejandro García (Universidad Nacional de

San Juan, CONICET)

Prof. Nélida De Grandis (Universidad Nacional de

Rosario)

Dr. Juan Bautista Leoni (Universidad Nacional de

Rosario, CONICET)

Dra. Amancay Martínez (Universidad Nacional de San

Luis)

Dra. Catalina Teresa Michieli (Universidad Nacional de

San Juan, CONICET)

Lic. Fernando Oliva (Universidad Nacional de Rosario)

Ing. Adrián Pifferetti (Universidad Tecnológica
Nacional Regional Rosario)

Dr. Mariano Ramos (Universidad Nacional de Luján, CONICET)

Dra. Ana María Rocchietti (Universidad Nacional de Rosario)

Dr. Daniel Schávelzon, (Universidad Nacional de Buenos

Aires, CONICET)

Dra. Carlota Sempé (Universidad Nacional de La Plata)

Dr. Mario Silveira (Universidad Nacional de Buenos Aires)

Dra. Silvia Simonassi (Universidad Nacional de Rosario)

Dra. Alicia Tapia (Universidad Nacional de Buenos Aires, Universidad Nacional de Luján)

Lic. Mónica P. Valentini (Universidad Nacional de Rosario)

Agrim. Benito Vicioso (Universidad Nacional de Rosario)

\section{Evaluaron este volumen}

Roberto Bárcena, María Teresa Boschin, Leonel Cabrera, Ulises Camino, María Rosa Carbonari, Carlos Ceruti, Horacio Chiavazza, Nicolás Ciarlo, Silvia Cornero, Eduardo Crivelli, Javier García Cano, Martín Gentinetta, María Laura Gili, Carlos Landa, Matilde Lanza, Melina Malandrino, Sebastián Pastor, Victoria Pedrotta, Josefina Piana,

Mercedes Podestá, Mariano Ramos, Daniel Schavelzon, Diana Tamburini, Mónica Therrien, Mónica Valentini y María Teresa Boschin

\section{Diseño y diagramación}

Eugenia Reboiro

(eugenia.reboiro@gmail.com)

\section{Curadoría}

Ana Rocchietti e Irene Dosztal

Foto de tapa: imagen del texto de Croce.

\section{Propietario responsable:}

Facultad de Humanidades y Artes, Universidad Nacional de Rosario Centro de Estudios de Arqueología Histórica

Entre Ríos 758. Rosario, provincia de Santa

Fe (2000). Argentina.

Telf.: +54 (0341) 4802670

E-mail: ceahunr@gmail.com

Decreto Ley 6422/57 de Publicaciones

Periódicas 


\section{Índice}

Editorial.

Arqueología histórica: programa de investigación y dimensiones epistemológicas...9 Ana María Rocchietti

De lozas, iglesias y machaqueños. Primeros pasos hacia una arqueología histórica en la cuenca norte del río desaguadero (La Paz, Bolivia).

Salvador Arano Romero

Identificación arqueológica de acciones militares en el campo de batalla de Cepeda, 1859.

Juan B. Leoni, Lucas H. Martínez, Cecilia Arias Morales, Daniela Cadenas,

Faustino Godoy, Mauro Ganem, María de la Paz Blanche y Héctor Meletta

Para una historia de la arqueología urbana en Buenos Aires (1848-1910) .59

Daniel Schávelzon y Francisco Girelli

Definiendo la cultura material colonial a partir de colecciones arqueológicas del Museo de La Plata.

Melina Bednarz, Julieta Penesis y Ana Igareta

La tangibilidad del conflicto: Arqueología del presente en las fronteras del norte santafesino....

Paula Del Río y Silvia Cornero

Arqueología histórica: evidencia material en el paisaje del establecimiento jesuita "La saladilla” (quebrada de Saladillo, La Rioja, Argentina). Juan Carlos Giuliano, Maira Ayelén Carrizo y María Soledad Gheggi

Graffiti ¿evidencia arqueológica o acto vandálico?. Camila Oliva

Arqueología histórica de los sistemas hidráulicos de la Hacienda

Jalpa de Cánovas, Guanajuato, México, y su relación con el sistema-mundo, Siglos XVIII-XIX

José Alberto Aguirre Anaya y Edgar Quispe Pastrana 
Paseo del bajo: una trinchera en la historia de Buenos Aires.................................143 Federico Martín Croce

Diferentes concepciones del patrimonio y su aplicación a la Casa Histórica de Tucumán también llamada Casa de la Independencia........................................155 Guillermo Etchevers

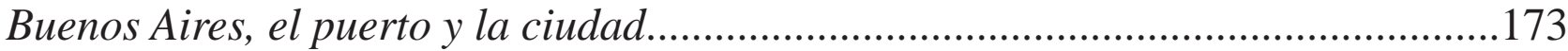
Mónica P. Valentini y Javier García Cano 


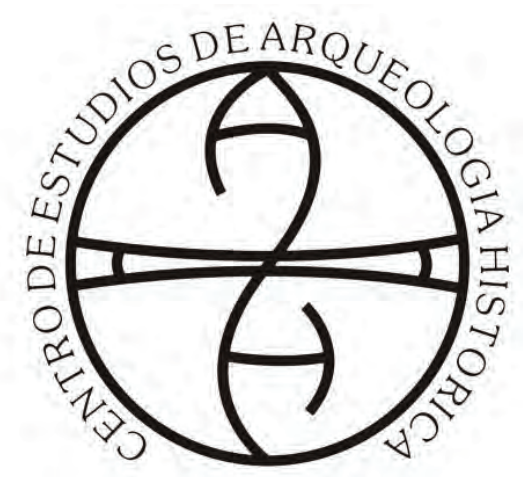

Centro de Estudios de Arqueología Histórica

Universidad Nacional de Rosario
Teoría y Práctica de la Arqueología Histórica

Latinoamericana | Año VIII. Vol. 8 | 2019

Revista del Centro de Estudios de Arqueología

Histórica, Facultad de Humanidades y Artes,

Universidad Nacional de Rosario

https://teoriaypracticaah.unr.edu.ar/index.php/index

ceahunr@gmail.com

ISSN en línea: 2591-2801

ISSN versión impresa: 2250-866X

\title{
GRAFFITI ¿EVIDENCIA ARQUEOLÓGICA O ACTO VANDÁLICO?
}

\author{
Camila Oliva*
}

\section{Resumen}

Este trabajo tiene como objetivo reflexionar respecto al carácter patrimonial de las prácticas de intervención cultural consistentes en la realización de graffiti y/o grabados a lo largo del último siglo. Para este propósito, se abordan tales manifestaciones, consideradas prácticas sociales de construcción de sentido, en contextos rurales y urbanos de la Provincia de Buenos Aires. En el primer caso, se trata de graffiti presentes en sitios arqueológicos prehispánicos del Área del Sistema Serrano de Ventania (sector sur del Área Ecotonal Húmedo Seca Pampeana); en el segundo caso se toman en consideración grabados emplazados en un sector del ejido urbano de la ciudad de Mar del Plata, -partido de General Pueyrredón ubicado en el sudeste bonaerense-. La disciplina arqueológica ha considerado escasamente a este tipo de registro como su objeto de estudio, mayoritariamente se ha subestimado su potencial histórico en relación al análisis de sus dimensiones simbólicas y enunciativas. En esta oportunidad se considera que la realización de graffiti constituye una práctica de representación social, situada espacial y temporalmente, cuyas dimensiones materiales y simbólicas pueden ser abordadas desde la Arqueología Histórica como fuente testimonial de especificidades históricas culturales. Por otra parte, constituye un tipo de patrimonio escasamente reconocido y problematizado. En este sentido, este trabajo intenta ser un aporte al reconocimiento de este tipo de registro como evidencia legítima de la cultura material de la historia de los pueblos.

Palabras clave: Patrimonio histórico, graffiti, contexto arqueológico.

\begin{abstract}
The objective of this work is to reflect on the patrimonial nature of cultural intervention practices consisting of the production of graffiti over the last century. For this purpose, such manifestations are considered, considered social practices of construction of meaning, in rural and urban contexts of the Province of Buenos Aires. In the first case, it is graffiti present in pre-Hispanic archeological sites of the Sistema Serrano de Ventania's area (southern sector of the Área Ecotonal Húmedo Seca Pampeana); in the second case, engravings placed in a sector of the city of Mar del Plata, district of General Pueyrredón located in

* Becaria Universidad Nacional de La Plata, Laboratorio de Análisis Cerámicos, Facultad de Ciencias Naturales y MuseoUNLP; Centro de Estudios Arqueológicos Regionales FHUMyAr Universidad Nacional de Rosario. Email: oliva.cami@ gmail.com; coliva@fcnym.unlp.edu.ar
\end{abstract}


the southeast of Buenos Aires, are taken into consideration. The archaeological discipline has scarcely considered this type of registry as its object of study, its historical potential has mostly been underestimated in relation to the analysis of its symbolic and enunciative dimensions. In this opportunity, graffiti is considered a practice of social representation, located spatially and temporally, whose material and symbolic dimensions can be addressed from the Historical Archeology as a testimonial source of historical cultural specificities. On the other hand, it constitutes a scarcely recognized and problematized type of heritage. In this sense, this work tries to be a contribution to the recognition of this type of registration as legitimate evidence of the material culture of the history of the peoples.

Keywords: Historical heritage, graffiti, archaeological context.

\section{Introducción}

En las últimas décadas, los enfoques de investigación en arqueología a nivel nacional e internacional han construido su objeto de estudio en torno a los graffiti como uno de los principales motivos de deterioro antrópico en monumentos y edificaciones históricas (Cardoso Arévalo, 2017); así como también una de las principales causas de destrucción de los sitios arqueológicos prehistóricos europeos (Carrera Ramírez, 2002) y americanos (Argüello García, 2006). Este tipo de práctica cultural ha sido abordada prácticamente de manera exclusiva en relación a su carácter intrusivo y disruptivo de la conservación de otras evidencias y patrimonios (e.g. sitios de carácter monumental tales como cuevas y aleros con arte rupestre, estructuras de piedras, entre otros) (Oliva et al., 2014; Sfeir et. al, 2013). Es en este marco que las dimensiones simbólicas, así como el carácter enunciativo y la propia historiografía de estas intervenciones han sido escasamente considerados por los estudios académicos desde la disciplina arqueológica.

En esta oportunidad se sostiene que la realización de graffiti constituye una práctica de representación social, situada espacial y temporalmente, cuyas dimensiones materiales y simbólicas deben ser abordadas desde la Arqueología Histórica. En virtud de ello, son considerados potenciales indicadores historiográficos atravesados por dimensiones tanto políticas como ideológicas, económicas y sociales (Benchimol, 1998; Nicora, 2016). ¿En qué consisten los graffiti históricos? ¿En qué casos es adecuado hablar de graffiti patrimoniales? ¿Qué actores los ejecutan a lo largo del tiempo? ¿Qué espacios son elegidos para su elaboración en contextos urbanos y rurales? ¿Qué se graffitea? ¿Cuáles son las intencionalidades de la práctica? ¿Qué técnicas son implementadas en su realización? ¿Cómo se relacionan las comunidades actuales con los sitios graffiteados? Son algunos de los interrogantes que guían esta investigación. El objetivo de este trabajo es reflexionar respecto al carácter patrimonial de las prácticas de intervención cultural consistentes en la realización de graffiti y/o grabados a lo largo del último siglo en áreas urbanas y rurales. Aquí se aplica el concepto de patrimonio entendido en su sentido constructivista, como una construcción social colectiva cuyo significado se relaciona a las memorias, las necesidades e intereses del presente, en el marco de los cuales diversas prácticas y bienes culturales pueden ser considerados patrimoniables (Prats, 2005). En este sentido, se propone discutir la categoría de graffiti y reflexionar acerca de su constitución como evidencia material arqueológica y como parte del patrimonio cultural histórico. 
Teoría y Práctica de la Arqueología Histórica Latinoamericana | Año VIII. Vol. 8 | 2019

ISSN en línea: 2591-2801 | ISSN versión impresa: 2250-866X

\section{¿Qué entendemos por graffiti?}

Los graffiti son considerados expresiones de importante capital social y cultural (Scharagrodsky, 2003), se tratan de un tipo de manifestación comunicativa efectuada con objetos punzantes, marcadores o pintura, en cualquier soporte urbano, realizadas con una clara intención transgresora (Blanco, 2003). Asimismo, se presentan como inscripciones en el espacio público no autorizadas legalmente aunque más o menos toleradas, asociadas principalmente a subculturas jóvenes (Kozak, 2005). De igual modo, constituyen prácticas de construcción de sentido en el paisaje, de modo que se posicionan como afirmaciones del derecho a estar en un lugar. En este sentido, el graffiti funciona como un tipo de marcador espacial que evidencia la interacción humana con su ambiente construido. N. García Canclini propuso en 1990 que se trata de un género constitucionalmente híbrido, ya que se caracteriza por ser un medio sincrético y transcultural que muchas veces afirma el territorio pero de un modo marginal, desinstitucionalizado, anónimo y efímero ignorando las relaciones entre lo privado y lo público, entre la vida cotidiana y lo político (Gracía Canclini, 1990; Scharagrodsky, 2003). De igual modo, C. Kozak asegura que "Se trata de una masa impresionante de inscripciones que abruman al ojo dispuesto a dar un sentido a la mirada. Están allí, tienen historia, se conectan con ciertos grupos sociales más que con otros, establecen territorios, formulan modos de experimentación del espacio urbano tanto para quienes los producen como para quienes los leen, plantean cantidades de preguntas...” (Kozak, 2005, p.2).

Algunos autores definen al graffiti en relación a su carácter ilegal, en este sentido el término graffiti es utilizado como sinónimo de una escritura o dibujo elaborado al margen de la legalidad y expuesto públicamente sobre superficies no diseñadas para tal fin (Petersen s.f). Otros destacan sus cualidades artísticas, caracterizándolo como un tipo de arte callejero, dialógico, el cual tiene como objeto establecer una semántica intertextual entre orden y anarquía (Gabbay, 2013). Siguiendo a M.V. Nicora, se considera graffiti a una multiplicidad de expresiones parietales que incluyen desde las formas pompeyanas o romanas, pasando por las pintadas que remiten a la política partidista y hasta lo conocido como estilo hip - hop o callejero (Nicora, 2016). Esta autora, sostiene que la amplísima gama de representaciones que se abren bajo este término produce confusión al momento de darle especificidad al objeto de estudio. Este trabajo propone problematizar el estudio de este tipo de evidencia desde la Arqueología Histórica, y abordarla como cualquier otra fuente documental. En este sentido, con un criterio puramente clasificatorio se propone dividir a los graffiti históricos en tres grandes grupos: un primer grupo denominado "graffiti centenarios" que incluye a aquellas manifestaciones que poseen una antigüedad igual o mayor a un siglo; un segundo grupo llamado "graffiti de temporalidad media" compuesto por aquellos graffiti que tienen una antigüedad superior a medio siglo, y finalmente "contemporáneos" a aquellos realizados con posteridad a la década de 1960.

\section{El graffiti como objeto de estudio académico: ¿agente destructivo o fuente de información?}

Los graffiti constituyen un tipo de expresión cultural muy diverso respecto a su apreciación por parte del universo académico. Algunos autores enfatizan que su importancia radica y depende del contexto político pictórico en el que se inserta dicha expresión (Gabbay, 2013), para otros es fundamental el criterio de su localización, en otras palabras si se trata de inscripciones sobre espacios públicos abiertos, expuestos a la mirada pública en las calles (e.g. sobre paredes, el pavimento, entre otros) o cerrados (e.g. escuelas, baños, cárceles). En el caso de los graffiti históricos se considera que sus dimensiones espaciales y temporales cobran especial importancia para diferenciar este conjunto heterogéneo de manifestaciones y evaluar su carácter patrimonial. 
Dentro de las investigaciones de las Ciencias Sociales se ha tendido a polarizar las opiniones respecto a estas manifestaciones. Desde uno de los extremos se han considerado a los graffiti como la principal causa de la obstrucción visual en los estudios de arte rupestre a través del tiempo, ya que debido a su presencia en estos sitios, proporciones significativas de las pinturas han desaparecido o se encuentran en condiciones de visibilidad diferencial lo cual dificultad su documentación, análisis y preservación (Oliva et al., 2016). En este sentido, algunos autores incluso han asociado a estas manifestaciones con la forma en que la sociedad representa su historia visual a través de un arte destructivo, siendo el graffiti una forma de terrorismo visual (Nikolova, 2013). Desde un enfoque opuesto, otros autores valoran a estas manifestaciones como testimonios materiales susceptibles de ser investigados. En este sentido, en la década de 1980 F. Blake propuso entender al graffiti como un material arqueológico, el cual podría ser estudiado de igual manera que otros tipos de registros arqueológicos tales como el arte rupestre (Blake, 1981). En esta línea se han realizado algunas aproximaciones a su análisis; a escala nacional se ha prestado especial importancia a las características formales de la escritura del graffiti. Entre éstas últimas puede mencionarse la relación entre el texto y el contexto, el desgaste de las superficies, y otros tipos de evidencias de deterioro; tal es el caso de la investigación realizada sobre los graffiti urbanos presentes en muros y elementos arquitectónicos de la ex iglesia y colegio de la Compañía de Jesús de Mendoza en la ciudad de Mendoza (Benchimol et al., 1998); los graffiti analizados en cuevas y aleros con arte rupestre en contextos rurales del área del Sistema Serrano de Ventania (Oliva et al., 2016; Oliva et al., 2015); o el análisis de las inscripciones verbales de la década de 1930 de una cueva en la localidad de Oyola (provincia de Catamarca), entendidos como elementos constitutivos en la conformación de los montajes policrónicos de este sitio(Quesada y Gheco, 2015).

\section{El graffiti desde una mirada legalista.}

El graffiti como práctica contemporánea social se encuentra dentro del marco de la ilegalidad; en este sentido es sancionada por diferentes ordenanzas de organismos de control municipal (Randrup y Ferraresi, 2009). Sin embargo, un panorama diferente lo presentan aquellos graffiti históricos que debido a su profundidad temporal (centenarios y de temporalidad media) se posicionan en el presente como evidencias materiales óptimas del pasado. De esta manera, a algunas de estas manifestaciones -en el marco jurídico de la Ley Nacional 25.743 adoptada por la Provincia de Buenos Aires- se les debe atribuir un carácter patrimonial debido a su condición testimonial de la historia contemporánea bonaerense. La Ley 25.743 expresa que forman parte del patrimonio arqueológico las "cosas muebles e inmuebles o vestigios de cualquier naturaleza” que puedan proporcionar información sobre los grupos socioculturales que habitaron el país desde épocas precolombinas hasta épocas históricas recientes (Artículo 2 Ley 25.743). Cabe destacar el significativo potencial testimonial de aquellas inscripciones centenarias de fines de siglo XIX y principios de siglo XX, que forman parte de las evidencias arqueológicas que da cuenta de los usos espaciales de determinados grupos sociales. A modo de ejemplo, puede mencionarse las inscripciones de la cueva Oyola, las cuales se cree que fueron realizadas por un arriero de ganado, motivo por el cual los autores sugieren que ese sitio se trataba de un refugio y/o un puesto de pastoreo (Quesada y Gheco, 2015); otro ejemplo son los graffiti centenarios de la ciudad de Mar del Plata, correspondientes a las familias aristocráticas que veraneaban en esta ciudad en las primeras décadas del siglo pasado (Oliva y Moscardi, e.p.). Asimismo, los graffiti verbales consistentes en apellidos de familias específicas, aportan elementos para las reconstrucciones biográficas, los estudios poblacionales y la indagación de la historia de la formación de las localidades y poblados actuales. Otro caso de particular interés, son las inscrip- 
ciones que remiten a nombres propios de instituciones históricas, tales como establecimientos educativos (e.g. graffiti verbal de temporalidad media que nombra al Colegio Nacional en el sitio Varese (Oliva y Moscardi, e.p.)), o los graffiti contemporáneos que arrojan luz sobre episodios concretos de la historia nacional. Entre estos últimos se encuentran aquellos vinculados a la reconstrucción histórica de los períodos de terrorismo de Estado en la última dictadura cívico militar, por ejemplo el análisis de graffiti presentes en el Centro Clandestino La Base de Santa Lucía, la cual funcionó entre el año 1975 y 1982 en el departamento de Monteros provincia de Tucumán (Cattaneo, 2015), entre otros casos. Por todo lo expresado, es adecuado sostener el carácter patrimonial que los graffiti históricos poseen en relación a su condición de soportes de memoria. En este sentido, se encuentran bajo tutela del Estado nacional, quién deberá adoptar las medidas tendientes a su preservación, investigación y divulgación, tal como se expresa en el artículo $4^{\circ}$ inciso a de la mencionada Ley.

Es importante señalar que la asignación de "patrimoniable” a este tipo de registro, en muchos casos resulta conflictiva, ya que la ejecución de graffiti sobre otros bienes patrimoniales ocasiona daños irreversibles y pérdida de información valiosa para la disciplina arqueológica y para la memoria histórica de pueblos que en muchos casos ya no se encuentran presentes (i.e. arte rupestre de grupos cazadores recolectores del Sistema Serrano de Ventania).

\section{Metodología}

En esta oportunidad, el análisis presta especial importancia a las similitudes y desemejanzas entre aquellas manifestaciones rurales y urbanas en el marco de su contextualización historiográfica. En este sentido, el trabajo analítico permite considerar a las intervenciones culturales en su relación directa con las identidades colectivas regionales, siendo una de las formas de abordar la manera en que las poblaciones rurales y metropolitanas se han vinculado con el territorio y su patrimonio durante el último siglo. Un factor trascendental a destacar es la multiplicidad del conjunto de graffiti analizados, en relación a una serie de variables tales como: su ubicación espacial dentro del sitio, la preexistencia o no de evidencia de ocupación humana previa en el soporte a ser graffiteado, el reconocimiento por parte del ejecutor de otro tipo de manifestaciones culturales precedentes, la cronología de la manifestación- la cual es posible conocer debido a que un gran porcentaje de graffiti poseen inscriptas fechas calendáricas que forman parte del registro-, entre otras.

Para cumplir con el objetivo propuesto se llevó a cabo en primera instancia la sistematización del registro fotográfico y documentado de los graffiti relevados y analizados con anterioridad en campañas previas (Oliva et al., 2016, Oliva y Oliva, 2018; Oliva y Moscardi, 2018). Posteriormente, se realizó la individualización de cada registro en unidades de composición independientes para analizar su contenido semántico, medio ejecutor, contexto (si se encuentra ejecutado sobre un soporte rocoso virgen, o en caso contrario sobre motivos rupestres), y qué agentes de deterioro (físicos, biológicos y antrópicos) se encontraban perjudicando la conservación de los graffiti arqueológicos en cada tipo de contexto (rural y urbano).

\section{Área de estudio}

Como se ha mencionado en apartados previos, este trabajo busca reflexionar respecto a los graffiti presentes en contextos urbanos y rurales de la provincia de Buenos Aires. Con este fin se toma en consideración a aquellas manifestaciones presentes en dos sectores de esta provincia. La fundamentación 
del área se basa en una serie de argumentos que a continuación se desarrollan. Para optimizar el estudio comparativo, se decidió trabajar en dos secciones de la provincia alejadas geográficamente entre sí, de este modo la ubicación geográfica fue una variable crucial a ser considerada. En este sentido, las áreas estudiadas debían estar ubicadas a una distancia igual o superior a los $300 \mathrm{~km}$. En virtud de ello, se escogió el Sistema Serrano de Ventania ubicado en el sudoeste bonaerense y el Sistema de Tandilia emplazado en el centro y sudeste de la provincia (Figura 1). Además se priorizó que presentaran características ambientales diferenciales, siendo un caso un ambiente serrano (Ventania) y en el otro, marítimo (últimas estribaciones de las sierras presentes en la ciudad de Mar del Plata). Por otra parte, respetando el objetivo principal, se decidió trabajar con estas regiones por sus importantes contrastes en relación a la magnitud de la densidad poblacional, el número y dimensión de las construcciones edilicias, los flujos migratorios (permanente y de corta duración), y la actividad económica. De esta manera, se resolvió investigar los graffiti del Sistema de Ventania, particularmente de los partidos de Tornquist y Saavedra, ambos partidos constituidos por pequeñas localidades rurales, y aquellos de la ciudad portuaria de Mar del Plata, estando esta en un contexto plenamente urbano (Figura 1).

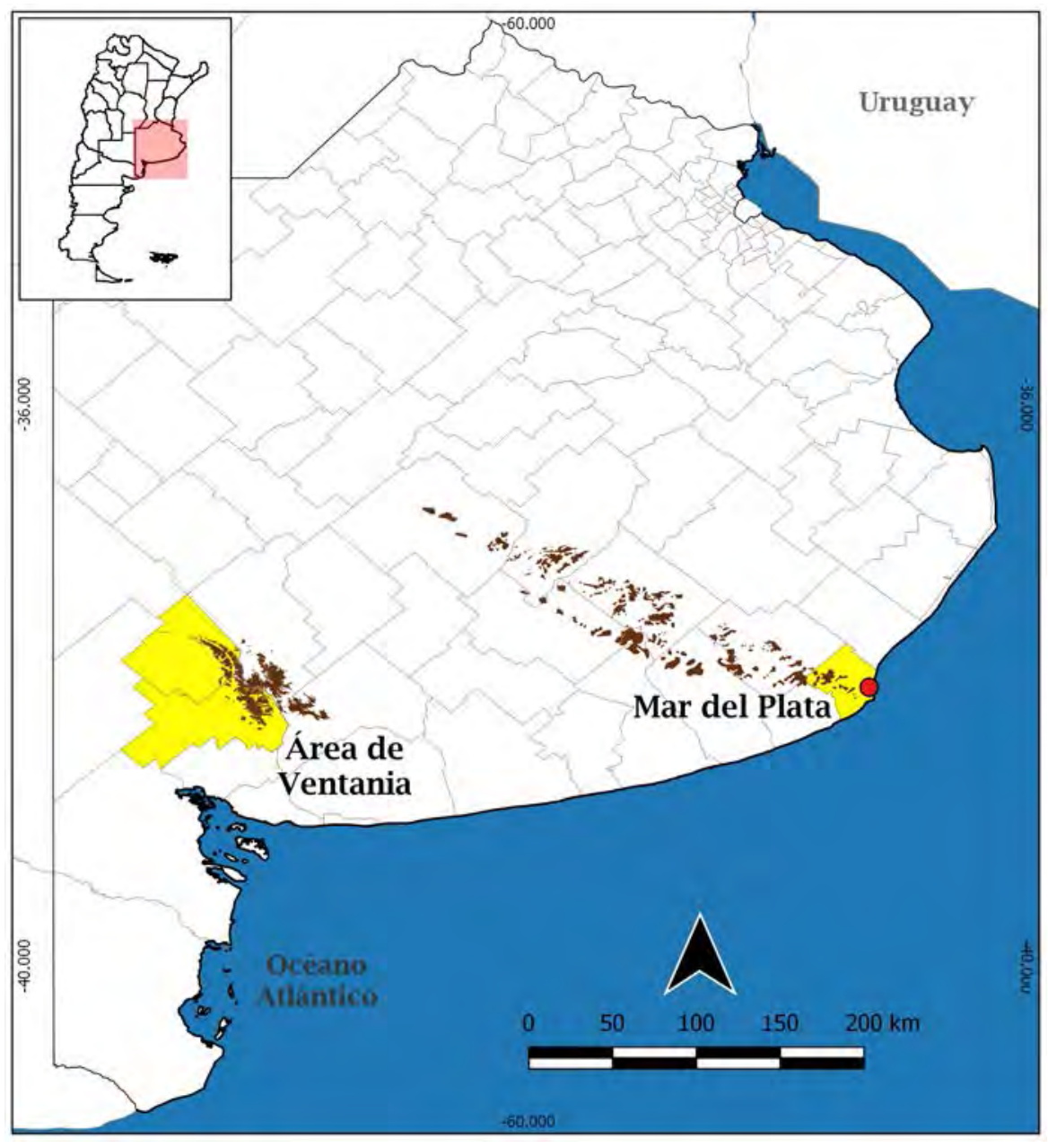

Figura 1. Área de estudio. En amarillo se encuentran resaltados los partidos de Saavedra, Tornquist y General Pueyrredón. Programa utilizado QGIS 2.14.01. 
Teoría y Práctica de la Arqueología Histórica Latinoamericana | Año VIII. Vol. 8 | 2019

ISSN en línea: 2591-2801 | ISSN versión impresa: 2250-866X

\section{Contexto urbano.}

El primer testimonio de graffiti investigado en un área urbana data de 1904, éste fue registrado por José María Ramos Mejía en la ciudad de Buenos Aires, éste autor vinculo a los graffiti con la capacidad de agencia de individuos cuyo propósito era fragmentar el sistema materializado, a través de las letras consideradas grietas invisibles, en el cuerpo de la urbe (Gabbay, 2013). C. Kozak (2005), cuyas investigaciones se caracterizan por indagar los modos en que los graffiti participan en la construcción de los sentidos urbanos, destacó a un tipo particular que denominó “pintada política”. Este subgrupo, según la autora, existe desde antes de 1810, por ejemplo en las leyendas presentes en la Ciudad de Buenos Aires en contra del Virrey, la frase "las ideas no matan” que Sarmiento traduce del francés en su paso al exilio en 1845, entre otros casos reconocidos (Kozak, 2005).

Posteriormente, durante todo el siglo XX pero con mayor énfasis durante la década de los noventa, con el auge de los estudios hermenéuticos, estas manifestaciones urbanas fueron extensamente investigadas desde el campo de los estudios en comunicación, y la historia del arte, entre otras áreas disciplinares (Gándara, 2002; Branda, 2015). Desde estos enfoques los graffiti son considerados medios de expresión en contra del establishment político y artístico, portadores de denuncias testimoniales que buscan oponerse a la violencia que impone la ciudad. En virtud de ello, durante todo el siglo XX estas expresiones dejaron sus huellas como respuestas políticas a los sucesos de orden público (Gabbay, 2013).

\section{Sitio Varese: el caso marplatense.}

La ciudad de Mar de la Plata, se encuentra emplazada sobre la línea de costa del partido de General Pueyrredón en el sudeste bonaerense. Fue fundada en el año 1874 por Patricio Peralta Ramos, y actualmente la habitan 618.989 personas (según estadísticas oficiales del Gobierno Municipal de General Pueyrredón). Esta localidad, llamada tempranamente la Perla del Atlántico se convirtió en uno de los principales centros turísticos para los argentinos. En primera instancia fue la villa balnearia escogida por la aristocracia porteña, y posteriormente desde la década de 1940 comenzó a ser visitada por un flujo de turismo masivo vinculado a la clase obrera y trabajadora (Oliva y Moscardi, e.p.). Desde ese entonces recibe un alto número de visitas, principalmente durante el período estival. Se estima que 616.559 personas la frecuentan en promedio durante el período de un año (datos obtenidos del promedio de visitantes del año 2017 publicados en https://www.mardelplata.gob.ar/).

El sitio Varese se trata de un emplazamiento a cielo abierto (Figura 2), adyacente al Paseo Jesús Galindez, se encuentra ubicado entre la playa que lleva hoy su nombre (antiguamente llamada Playa de los Ingleses) en el sector central de la costa de la ciudad y el edificio del Torreón del Monje. Ésta edificación, construida en el año 1904, constituye uno de los íconos arquitectónicos más emblemáticos de la ciudad, por lo que se considera que puede haber funcionado como un importante atractor espacial para los transeúntes que visitaban el lugar. Se posee documentación histórica (ver Figura 3) que atestigua la presencia de una gran cantidad de graffiti en sus inmediaciones desde principios del siglo XX. Cabe mencionar, que un sector del sitio próximo al Torreón del Monje, conocido como Gruta Egarte fue dinamitado con posteridad a la creación del puerto y la escollera sur en 1909, en pos de la construcción del camino portuario de la ciudad.

Actualmente, el sitio Varese se compone de un conjunto de graffiti históricos, de los cuales hasta el momento se han registrado y analizado 60 grabados esgrafiados sobre soportes rocosos adyacentes al Torreón del Monje (Oliva y Moscardi, 2018). Estos bloques poseen grandes dimensiones y corresponden 
a la formación Balcarce pertenecientes al sistema serrano de Tandilia. Los graffiti relevados son en su totalidad verbales, particularmente nombres propios, firmas, y siglas en algunos casos asociados a motivos icónicos vinculados a la actividad marítima y el tema "amor", entre otros (Oliva y Moscardi e.p.). Estas manifestaciones dan cuenta de la expresión histórica cultural de más de un siglo de historia de la ciudad de Mar del Plata, encontrándose inscripciones tan antiguas como de principios de siglo XX (Figura 4). Entre los agentes de deterioro relevados se registraron la acción eólica y marina (erosión física), la presencia de algas y micro organismos por encima de los graffiti (biodeterioro), y la presencia de restos de fogones y basura, así como el desgaste producido por los transeúntes y desagües cloacales de la urbe (Oliva y Moscardi e.p.).
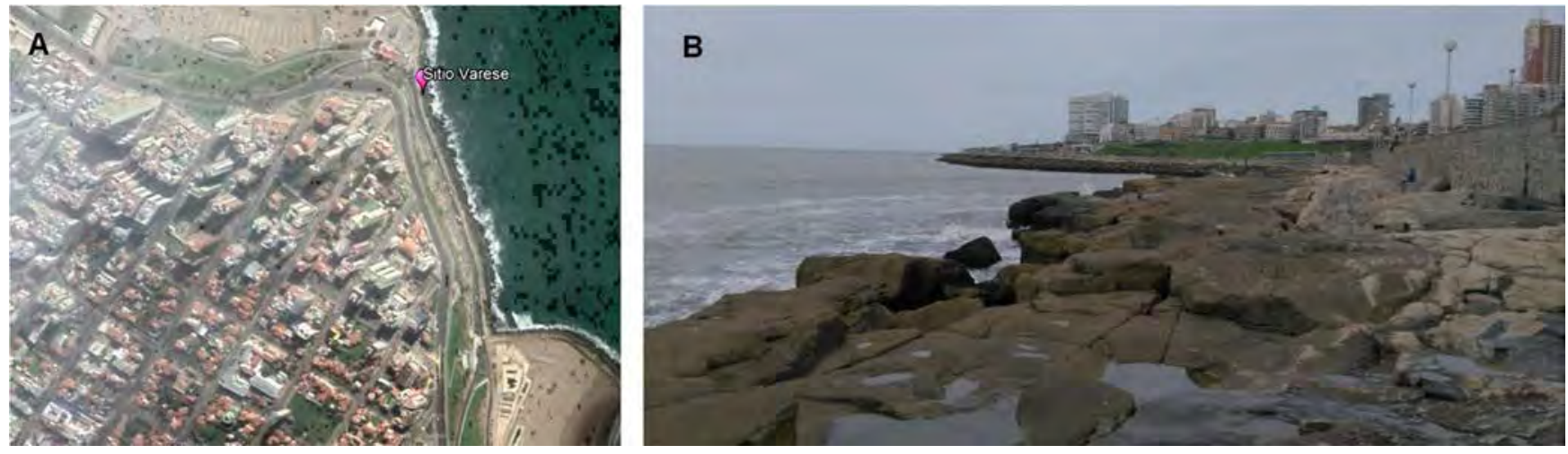

Figura 2. a) Vista aérea de la localización del sitio Varese, ciudad de Mar del Plata. Programa utilizado Google Maps. b) [Fotografía propia]. (Mar del Plata. 2017).Sitio Varese, se observan los bloques de la formación Balcarce, sistema serrano de Tandilia.
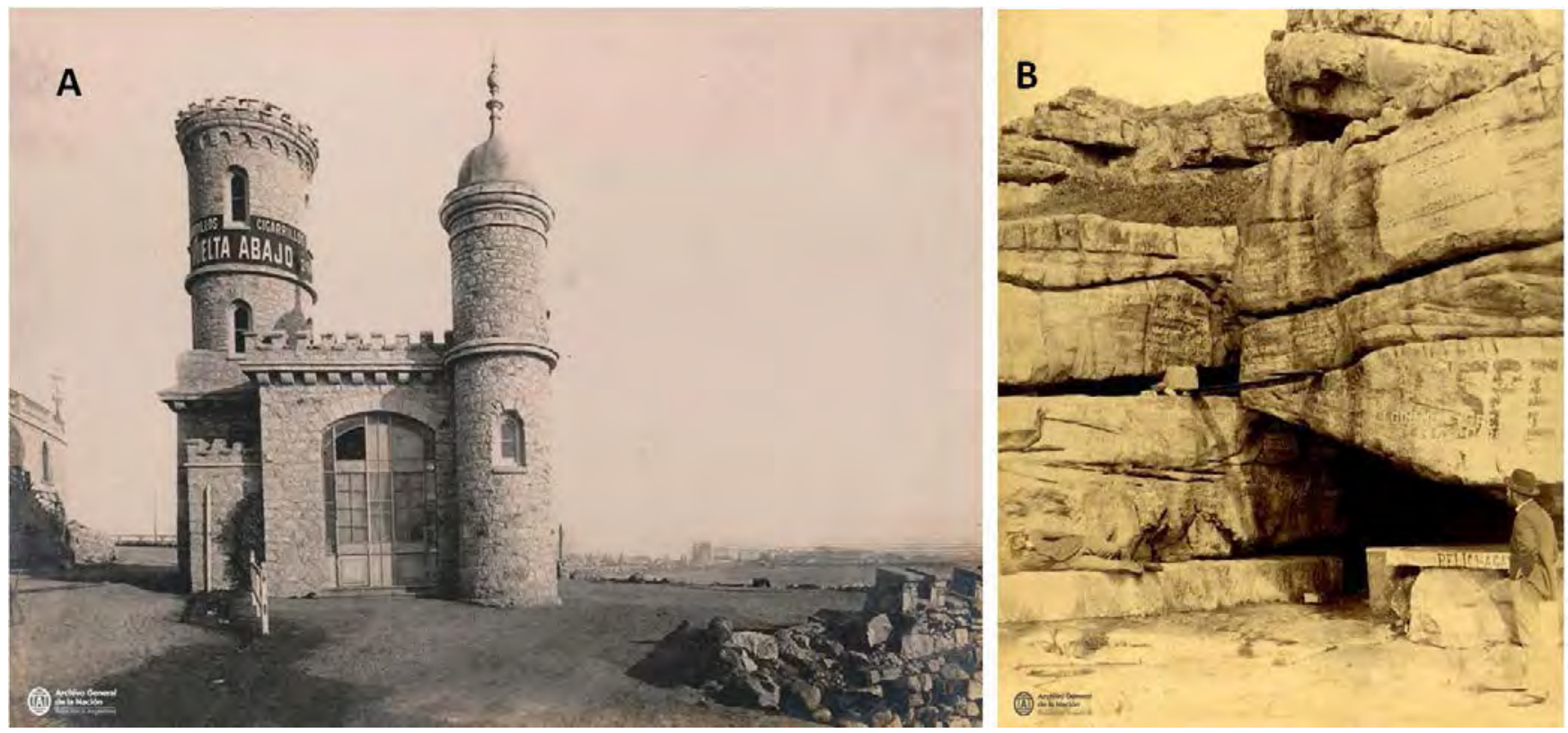

Figura 3. Izquierda: [Fotografía Archivo General de la Nación]. (Mar del Plata. 1910). AGN_DDF_Fondo Aficionados/ Caja 26, inv: 214210. Se observa el Torreón del Monje de Mar del Plata en 1910. Derecha: [Fotografía Archivo General de la Nación]. (Mar del Plata. 1910). AGN_DDF_Fondo Aficionados/ Caja 3, inv: 213155. Se observa el sector de la Gruta Egaña, en el año 1910. 


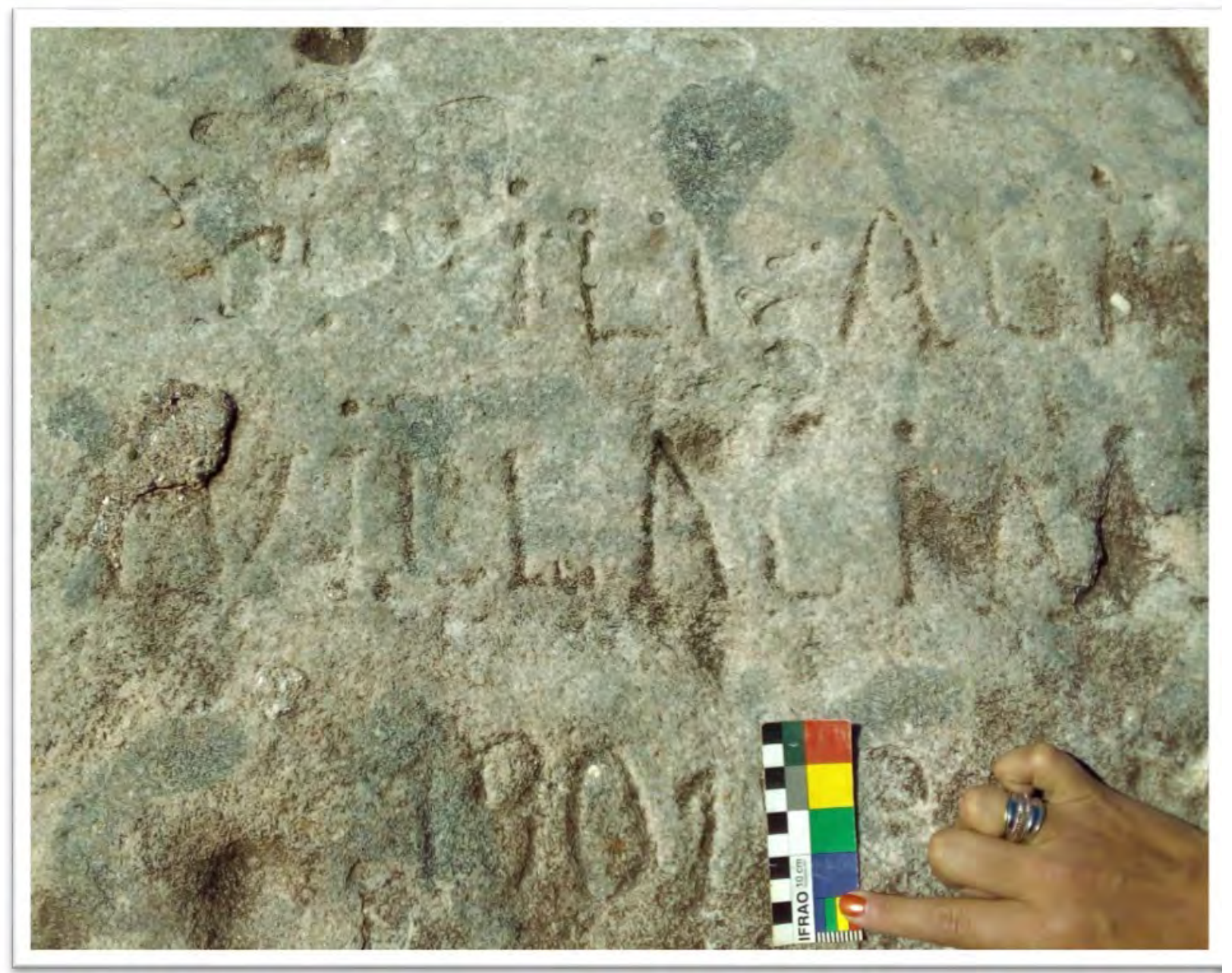

Figura 4. [Fotografía propia]. (Mar del Plata. 2018). Grabado histórico del sitio Varese, ciudad de Mar del Plata.

Por otra parte, cabe destacar que este sitio recibió una declaración a nivel provincial como yacimiento paleontológico excepcional debido a la presencia de la traza fósil de más de cinco millones de años de historia paleontológica (expediente 2240 16/17). Asimismo, se encuentra protegido por ordenanzas municipales (ordenanza 22325/2015 y 9417/1994), las cuales declaran de interés patrimonial municipal las piezas y yacimientos arqueológicos y paleontológicos situados en el Partido de General Pueyrredón, tutelados por la legislación nacional y provincial.

\section{Contexto rural: graffiti del Sistema de Ventania.}

Por su parte, el Área del Sistema Serrano de Ventania constituye una de las tres serranías que sobresalen en el paisaje de llanuras que caracterizan a la Región Pampeana, siendo este el de mayor altitud dentro de la provincia de Buenos Aires. Se localiza en el sector sur del Área Ecotonal Húmedo Seca Pampeana, ubicada en el sudoeste de la provincia. Esta región se caracteriza actualmente por una fuerte 
actividad económica agrícola ganadera. En relación a la historia de su ocupación, fue poblada por sociedades cazadoras recolectoras desde hace al menos 6.000 años (Castro 1983). Las investigaciones en el área han dado como resultado el registro de más de 40 cuevas con arte rupestre (Oliva y Panizza, 2012), en este sentido se considera que se trata de un tipo de registro monumental recurrente en la zona. Las cuevas y aleros con pinturas relevadas se encuentran situadas dentro de los partidos bonaerenses de Tornquist y Saavedra. Actualmente, esta región se compone de pequeñas localidades agrícolas ganaderas, y cuenta con una población total de 30.223 habitantes (INDEC 2010).

La presencia de graffiti en el área se remonta a fechas tan tempranas como finales de siglo XIX. En el año 1884 Eduardo Ladislao Holmberg reconoció en el sitio Gruta de los espíritus (ubicado en el valle intraserrano, partido de Saavedra) graffiti próximos a las pinturas rupestres prehispánicas (Holmberg, 1884).

Se ha llevado el registro de 741 graffiti en 17 cuevas y aleros con arte rupestre (Figura 5). Seis de éstas (Alero Corpus Christi, Cueva del Toro, Cueva Parque Provincial Ernesto Tornquist (PPET), Cueva 3 y 4 PPET, y Cueva Florencio) se encuentran en áreas administradas por la Provincia de Buenos Aires, específicamente por el Organismo Provincial para el Desarrollo Sostenible (cinco en el Parque Provincial Ernesto Tornquist y una en la Reserva Natural Sierras Grandes). El resto se hallan dentro de campos de propiedad privada.

De las 741 manifestaciones relevadas, 664 se encuentran en un solo sitio (Gruta de los Espíritus). Éste fenómeno da cuenta de la superposición de graffiti, siendo las nuevas inscripciones fuente de deterioro de aquellas inscripciones previas. Otros agentes culturales de deterioro identificados son el descarte de basura actual y la realización de fogatas dentro o en inmediaciones de las cuevas, entre otros. Por otra parte, la acción de microorganismos, líquenes y musgos son recurrentes en este tipo de ambientes cerrados (cuevas) y han sido documentados extensamente (Gallego y Oliva, 2005; Gallego y Panizza, 2005).
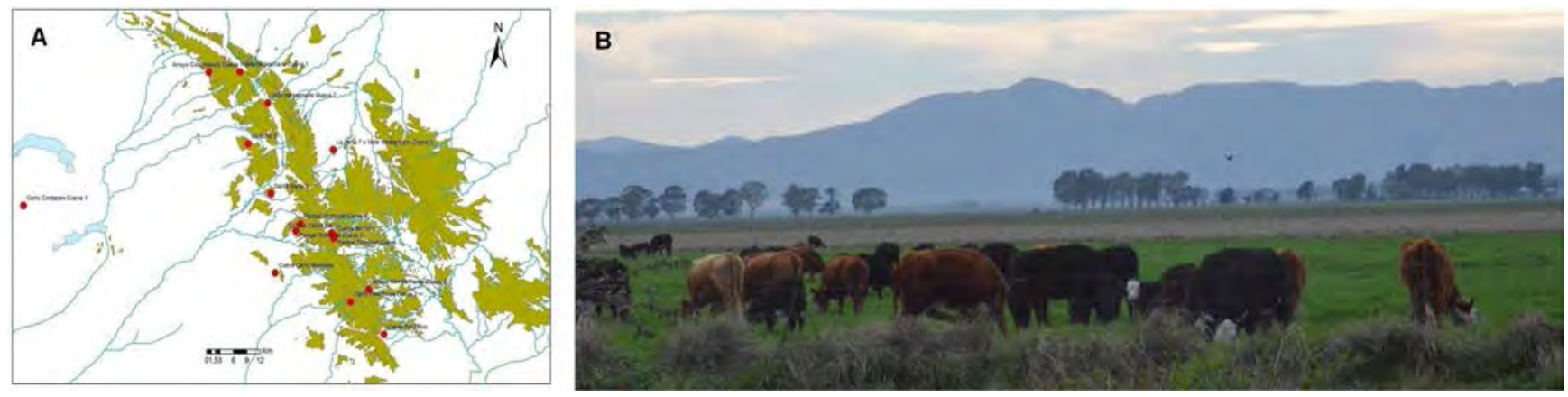

Figura 5. a) Representación de la ubicación de las cuevas y aleros con presencia de graffiti en el Sistema Serrano de Ventania. Programa utilizado QGIS 2.14.01. b) [Fotografía propia]. (Fotografía tomada desde la localidad de Dufaur, partido de Saavedra. 2018). Actividad rural agrícola ganadera, de fondo el Sistema Serrano de Ventania. 


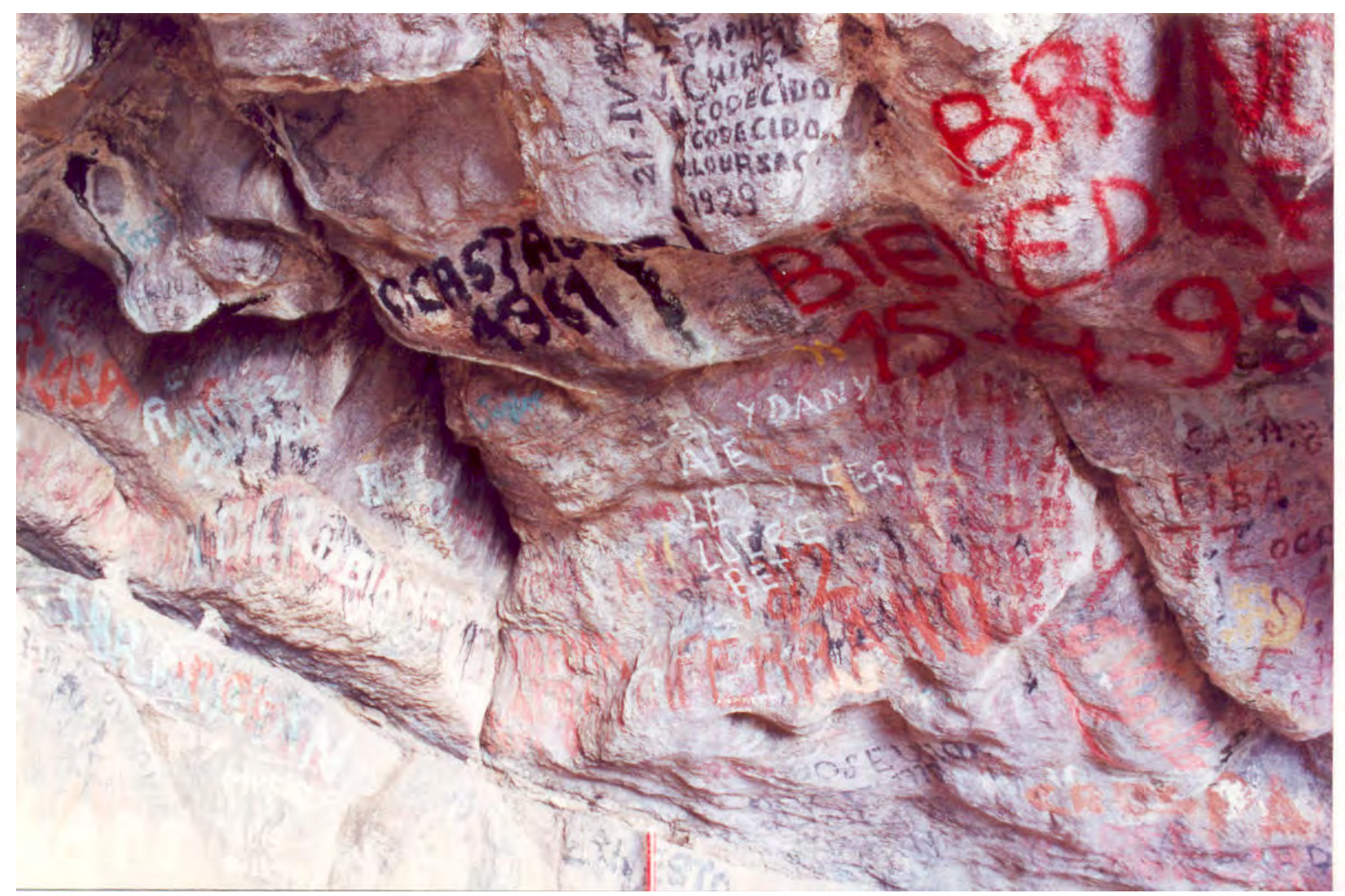

Figura 6. [Fotografía Fernando Oliva]. (Gruta de los Espíritus, parido de Saavedra. 2006). Panel de graffiti del sitio Gruta de los Espíritus, partido de Saavedra.

\section{Algunos resultados}

Para las área comprendidas en este trabajo se ha llevado un minucioso relevamiento y análisis de más de 800 graffiti (Oliva et al., 2016; Oliva et al., 2018); dentro de este conjunto se destacan manifestaciones de fines del siglo XIX para el caso del Sistema Serrano de Ventania e inicios de siglo XX para el caso de la ciudad de Mar del Plata. Cabe destacar la predominancia de graffiti verbales (736 del total) que constituyen firmas, nombres propios o siglas. Por tanto, se considera que hay un predominio del sentido testimonial, mediante referencias directas a las identidades personales de los autores de las inscripciones, y a las coordenadas temporales y en algunos casos espaciales de la inscripción. En este sentido, el autor C. Gabbay toma los aportes del filósofo y sociologo J. Baudrillard para afirmar que este tipo de manifestaciones constituyen un acto político al ocupar el espacio público, en el acto de reapropiación se busca simultáneamente restablecer la presencia individual y humana en el paisaje (Gabbay, 2013). En el caso de los grabados presentes en el sitio Varese se identifica una predominancia de las coordenadas espacio temporales de las inscripciones, en este sentido 46 de los graffiti relevados poseen una fecha calendárica asociada. Asimismo, estos grabados en su mayoría poseen inscripto el nombre de la ciudad "Mar del Plata”. Por el contrario, los graffiti del Sistema de Ventania la predominancia es sólo temporal, 
Teoría y Práctica de la Arqueología Histórica Latinoamericana | Año VIII. Vol. 8 | 2019

ISSN en línea: 2591-2801 | ISSN versión impresa: 2250-866X

excepcionalmente aparece el nombre de alguna localidad ajena a los partidos comprendidos en el área de estudio (e.g. Coronel Suárez).

Tabla 1. Graffiti relevados en el Área de estudio.

\begin{tabular}{lllll}
\hline & $\begin{array}{l}\text { Verbales } \\
\text { (Firmas/Nombres } \\
\text { propios) }\end{array}$ & $\begin{array}{l}\text { Fechas } \\
\text { Asociadas }\end{array}$ & $\begin{array}{l}\text { Motivos } \\
\text { iconográficos } \\
\text { asociados }\end{array}$ & $\begin{array}{l}\text { Verbales } \\
\text { (frases, } \\
\text { insultos) }\end{array}$
\end{tabular}

Por otra parte, ambos contextos arqueológicos se encuentran emplazados en áreas frecuentemente visitadas por agentes turísticos locales y no locales. Asimismo, comparten la característica de ser fácilmente perceptibles dentro del paisaje circundante ya que se encuentran en áreas elevadas y altamente atractivas. Estas características los convierten en lugares mayormente susceptibles de ser identificados por las poblaciones. Además, en ambos contextos se encuentran presentes agentes naturales vírgenes, en un caso el paisaje serrano y en el otro el mar, motivo por el cual se considera que la presencia de graffiti marca una intencionalidad de antropomorfización del paisaje.

Respecto a la reocupación de los espacios, la superposición de graffiti es entendida como la yuxtapoción de evidencias de actividad humana, lo cual permite pensar a estos sitios como palimpsestos de manifestaciones testimoniales de la historia humana bonaerense. Las diferentes capas conviven en un sitio de manera policrónica. En algunos casos se trata de una coexistencia altamente conflictiva, como el caso de los graffiti presentes en sitios con arte rupestre. Sin embargo, todas las “capas históricas” de evidencia forman parte constitutiva del sitio arqueológico. Cabe destacar que en el caso marplatense algunos de los grabados y graffiti llevados a cabo en soportes rocosos podrían estar situados sobre sitios de microorganismos paleontológicos; lo cual complejiza aún más la trama de relación entre los diversos registros del sitio.

En relación a su conservación los grabados del sitio Varese a pesar de estar sometidos a condiciones de deterioro físicas y ambientales con mayor impacto directo (erosión eólica y marina) poseen un mayor grado de conservación que los del Área de Ventania que se encuentran dentro de cuevas y abrigos. Esto puede estar fundamentado en la naturaleza de los grabados y su alto grado de perdurabilidad. Se trata de inscripciones profundas sobre la roca, en los cuales se utilizaron herramientas como cinceles para su elaboración. Contrariamente, en el área de Ventania, salvo la excepción de un conjunto de cuatro graffiti de la Cueva 3 arroyo Napostá, denominados “grabados profundos” debido a la intencionalidad de la técnica implementada por sus ejecutores (Oliva y Oliva, 2018), los graffiti consisten en su totalidad en pintadas 
Teoría y Práctica de la Arqueología Histórica Latinoamericana | Año VIII. Vol. 8 | 2019

ISSN en línea: 2591-2801 | ISSN versión impresa: 2250-866X

o rayados ocasionales con la materia prima disponible en el lugar. Por este motivo, la vida media de éstos últimos es más corta que aquellos preparados para su durabilidad. Como se ha expresado en apartados previos, los graffiti constituyen un tipo de manifestación conflictiva por su carácter disruptivo de otros patrimonios; sin embargo su alta representatividad y buena conservación lo posicionan como un tipo de evidencia arqueológica sumamente útil para el estudio de la historia contemporánea de la sociedad.

\section{Consideraciones finales}

Si se considera en términos amplios a la Arqueología Histórica como la especialidad dedicada a la investigación del pasado reciente, que en el caso latinoamericano cubre el rango cronológico iniciado con la invasión de los europeos al continente americano, por intermedio de la evidencia material producto de sus actividades y de otras fuentes de información tales como documentos escritos, pinturas, fotografías y registros orales (Landa y Ciarlo, 2016), la realización de graffiti constituye una práctica de representación social, situada espacial y temporalmente, cuyas dimensiones materiales y simbólicas, pueden y deben ser abordadas por este campo disciplinar. Este tipo de registro se coloca, junto a otras evidencias de época, como una fuente escrita susceptible de análisis, capaz de ser contrastable con otras fuentes documentales. Tal es el caso de las fotografías históricas utilizadas para la contextualización historiográfica de los graffiti del sitio Varese (ver Figura 3 B), o el relato de E.L. Holmberg sobre estas inscripciones en la Gruta de los Espíritus del Sistema Serrano de Ventania. Por otra parte, esta evidencia aporta elementos para dar cuenta de la dinámica de los procesos de construcción de paisajes culturalmente significativos, ya que la selección de los espacios con presencia de graffiti no resulta fortuita. En este sentido, los actores asumen una incidencia directa sobre la resignificación del territorio en el que viven y cargan simbólicamente. Se considera que la actualización histórica de estos espacios constituye un patrimonio vivo, que cobra sentido dentro de la red de significación social atribuida a estos espacios en una época que responde a un contexto histórico y espacial específico.

Es importante señalar que estudios de este tipo adquieren relevancia cuando se entiende que no hay discursos autorreferenciales, los graffiti verbales no son sólo firmas, sino que como menciona M.V. Nicora (2016), en todos los casos se trata de relaciones entre el discurso que es objeto de análisis y sus condiciones de producción, que son siempre históricas y tienen una existencia material encarnada en enunciados (Nicora, 2016). En síntesis, se trata de un tipo de evidencia material que consigue ser "patrimoniable”, debido a las valoraciones comunitarias en relación a su carácter de práctica social sostenida en el tiempo. Asimismo, la academia como parte de la sociedad, aprecia las connotaciones testimoniales que pueden hacerse a partir de este registro. Finalmente, se sostiene que el estudio de los palimpsestos de sitios con graffiti, pueden generar aportes a la conceptualización de patrimonio integral de una comunidad, considerado como una de las formas de la memoria colectiva que engloba la suma de manifestaciones de la acción humana y que constituyen la base de diferenciación de cada sociedad (Gili, 2007).

\section{Agradecimientos}

A la Universidad Nacional de La Plata por financiar esta investigación a través del otorgamiento de una beca doctoral. A M.C. Panizza y F.W.P. Oliva por su compromiso y participación activa a lo largo de los años en el relevamiento de los graffiti del Área de Ventania. A todos los dueños de establecimientos privados que permitieron realizar estos relevamientos. Finalmente, a B. Moscardi por su interés, dedicación y empeño en el estudio de la historia de Mar del Plata. 
Teoría y Práctica de la Arqueología Histórica Latinoamericana | Año VIII. Vol. 8 | 2019

ISSN en línea: 2591-2801 | ISSN versión impresa: 2250-866X

\section{Referencias bibliográficaas}

ARCHIVO GENERAL DE LA NACIÓN

ARCHIVO GENERAL VILLA MITRE

ARGÜELLO GARCÍA, P. M. (2006). El marco cultural de la restauración del arte rupestre: notas sobre un caso de estudio. Revista Virajes (8), 321-339.

BLAKE, F. C. (1981). Graffiti and racial insults: the archaeology of ethnic relations in Hawaii. En R. Gould y M. Schiffer Michael (comps.), Modern material culture: the archaeology of us (pp. 8898). Nueva York, Estados Unidos: Academic Press.

BENCHIMOL, S.; ALEVA, M.; GARCÍA, C.; GUIÑAZÚ, L. y ALMAZÁN, A. . (1998). Los grafitos de San Francisco: Relevamiento e Interpretación. En D. Schávelzon (Ed.) Las Ruinas de San Francisco (ex jesuitas), Arqueología e historia (pp 225-273). Mendoza, Argentina: Ed. Tintar.

BLANCO, J. M. (2003). De cara a la pared: graffiti alternativo y conciencia ciudadana. En I. Cornejo Portugal Texturas urbanas: comunicación y cultura (pp 229-264). Ciudad de México, México: Editorial Fundación Manuel Buendia.

BRANDA, M.J. (2015). Ciudad, imagen y comunicación: Comunicación visual en el espacio público. Arte e Investigación (11), 27-34.

CARDOSO ARÉVALO, K. (2018). Análisis comparativo de inventarios fotográficos. El uso de la información y la fotografía histórica en la gestión y conservación del patrimonio. Arte y Sociedad, Revista Investigación (14), 79-94.

CARRERA RAMÍREZ, F. (2002). Sugerencias para la preservación del patrimonio megalítico. MINIUS (9), 47-70.

CASTRO, A. (1983). Noticia preliminar sobre un yacimiento en Sierra de la Ventana, Sierras Australes de la Pcia. de Buenos Aires. Relaciones 15 (N.S.), 91-107.

EXPEDIENTE 2240 16/17 Declaración de interés provincial a la ciudad de Mar del Plata como yacimiento paleontológico excepcional.

CATTANEO, C. (2015). Memorias de la represión en los muros. La base militar de Santa Lucía. Dpto. Monteros, Tucumán (1975-1982). La zaranda de ideas, 13(2), 91-106. Recuperado de http://www. scielo.org.ar/scielo.php?script=sci_arttext\&pid=S185312962015000200003\&lng=es\&tlng=es.

GABBAY, C. (2013). El fenómeno posgrafiti en Buenos Aires. AISTHESIS (54), 123-146.

GILI, M. L. (2007). El conflicto y la tensión manifestante en los códigos de ética de los arqueólogos por legislar sobre los bienes culturales. En E. Olmedo y F. Rivero (comps.) Debates actuales en Arqueología y Etnohistoria. Publicación de las Vy VI Jornadas de Investigadores en Arqueología y Etnohistoria del Centro-Oeste del País (pp 343-356). Río Cuarto, Argentina: Universidad Nacional de Río Cuarto.

GÁNDARA, L. (2002). Graffiti. Buenos Aires: Argentina: Eudeba. 
Teoría y Práctica de la Arqueología Histórica Latinoamericana | Año VIII. Vol. 8 | 2019

ISSN en línea: 2591-2801 | ISSN versión impresa: 2250-866X

GARCÍA CANCLINI, N. (1990). Culturas Híbridas. Estrategias para entrar y salir de la modernidad. Distrito Federal, México: Grijalbo.

GALLEGO, M. y OLIVA, F. (2005). Evaluación de agentes de deterioro biológicos y culturales en cuevas y abrigos rocosos del Sistema Serrano de Ventania, Provincia de Buenos Aires. Revista de la Escuela de Antropología (11), 133-148.

GAlleGO, M. y PANIZZA, M. C. (2005). Arte rupestre del Sistema de Ventania (Pcia. de Buenos Aires). Estudio y monitoreo de los agentes naturales de deterioro. Libro de resúmenes del IV Congreso de Arqueología de la Región Pampeana Argentina. p. 159. Bahía Blanca.

HOLMBERG, E. (1884). La Sierra de Curá-Malal (Currumalan). Informe presentado al Excelentísimo Señor Gobernador de la provincia de Buenos Aires, Dr. Dardo Rocha. Buenos Aires. 81 pp.

KOZAK, C. (2005). Graffitis argentinos: Letra joven, letra urbana. Encrucijadas (3). Universidad de Buenos Aires. Disponible en el Repositorio Digital Institucional de la UBA. Repositoriouba.sisbi. uba.ar.

LANDA, C.G. y CIARLO, N. C. (2016). Arqueología histórica: especificidades del campo y problemáticas de estudio en Argentina. Quehaceres (3), 96-120.

LEY PROVINCIAL 25.743 de Protección del Patrimonio Arqueológico y Paleontológico (2003).

NICORA, M. V. (2016). Graffiti: ¿Caos o self? En F. Oliva, A.M. Rocchietti y F. Solomita(eds.) Imágenes Rupestres lugares y regiones (pp.71-82). Rosario, Argentina: FHUMyAR.

NIKOLOVA, L. (2013). Graffiti and archaeological art as paradox of culture and as values. Papers XXV Valcamonica Symposium, 1-4.

OLIVA, C. y MOSCARDI, B. e.p. Grabados históricos en la costa de Mar del Plata ¿Patrimonio Arqueológico? Aceptado para ser publicado en el Volumen especial de las VI Jornadas de Patrimonio Arqueológico y VII Jornadas de Patrimonio Paleontológico de la Provincia de Buenos Aires. Miramar, Argentina.

OLIVA, C. y MOSCARDI, B. (2018). Grabados históricos en la costa de Mar del Plata ¿Patrimonio Arqueológico? En: Actas de Libro de resúmenes de las VI Jornadas de Patrimonio Arqueológico y VII Jornadas de Patrimonio Paleontológico de la Provincia de Buenos Aires. Pp. 19. Miramar, Argentina.

OLIVA, F. y PANIZZA, M. C. (2012). Primera aproximación a la Arqueología Monumental del Sistema Serrano de Ventania, Pcia. de Bs. As. Anuario de Arqueología (4), 161-180.

OLIVA, F.; PANIZZA M. C y DEVOTO, M.G. (2014). Transformaciones naturales y culturales sobre el patrimonio arqueológico monumental del Sistema Serrano de Ventania. Reunión sobre Biodeterioro y Ambiente de la Provincia de Buenos Aires, 1-6.

OLIVA, F.; PANIZZA M. C y OLIVA C. (2016). Los graffti del área de Ventania: Expresiones contemporáneas de los usuarios del arte rupestre. En F. Oliva, A.M. Rocchietti y F. Solomita (eds.) Imágenes Rupestres lugares y regiones (pp. 343-356). Rosario, Argentina: FHUMyAR. 
PRATS, L. (2005). Concepto y gestión del patrimonio local. Cuadernos de Antropología Social (21), 17-35.

OLIVA, F. y OLIVA, C. 2018. Discursos, graffiti e identidades históricas del Sistema Serrano de Ventania, provincia de Buenos Aires (sector sur del Área Ecotonal Húmedo-Seca Pampeana). Revista de Arqueología Histórica Argentina y Latinoamericana Dossier “Arqueología Histórica Argentina. Situación y perspectivas” (12), 1164-1188.

ORDENANZA MUNICIPAL PARTIDO DE GENERAL PUEYRREDÓN 9417 año 1994.

ORDENANZA MUNICIPAL PARTIDO DE GENERAL PUEYRREDÓN 22325 año 2015.

PETERSEN, E. (s/f). El graffiti en Buenos Aires. Recuperado en http://www.elportaldemexico.com/arte/ artesplasticas/graffiti.htm

QUESADA, M. y GHECO, L. (2015). Tiempos, cuevas y pinturas. Reflexiones sobre la policronía del arte rupestre de Oyola (provincia de Catamarca, Argentina). Relaciones de la Sociedad Argentina de Antropología XL (2), 455-476.

RANDRUP, M. y FERRARESI, F. (2009). El graffti tiene la palabra (tesis de licenciatura). Facultad de Periodismo y Comunicación Social de la Universidad Nacional de La Plata, La Plata, Argentina.

SCHARAGRODSKY, P. (2003). Los graffitis y los cantitos futboleros platenses (o acerca del proceso de configuración de diversas masculinidades). Revista Oficios Terrestres Año IX, (13), 161-174.

SFEIR, A.; OLIVA, C.; DEVOTO, M. G. (2013). Distribución del registro monumental presente en el Sistema Serrano de Ventania y su relación con las poblaciones actuales. Anuario de Arqueología (5) 427-444.

www.mardelplata.gob.ar/

Recibido: 14 de febrero de 2019

Aceptado: 02 de junio de 2019 